\title{
M-Degrees of Quadrangle-Free Planar Graphs
}

\author{
Oleg V. Borodin, ${ }^{1}$ Alexandr V. Kostochka, ${ }^{1,2}$ \\ Naeem N. Sheikh, ${ }^{2}$ and Gexin $\mathbf{Y u}^{\mathbf{3}}$ \\ ${ }^{1}$ SOBOLEV INSTITUTE OF MATHEMATICS \\ NOVOSIBIRSK 630090, RUSSIA \\ E-mail: brdnoleg@math.nsc.ru \\ ${ }^{2}$ DEPARTMENT OF MATHEMATICS \\ UNIVERSITY OF ILLINOIS \\ URBANA, ILLINOIS 61801 \\ E-mail:kostochk@math.uiuc.edu; nsheikh@math.uiuc.edu \\ ${ }^{3}$ DEPARTMENT OF MATHEMATICS \\ VANDERBILT UNIVERSITY \\ NASHVILLE, TENNESSEE 37240 \\ E-mail: gexin.yu@vanderbilt.edu
}

Received May 25, 2007; Revised May 27, 2008

Published online 30 July 2008 in Wiley InterScience(www.interscience.wiley.com).

DOI 10.1002/jgt.20346

\begin{abstract}
The M-degree of an edge $x y$ in a graph is the maximum of the degrees of $x$ and $y$. The $M$-degree of a graph $G$ is the minimum over $M$-degrees of its edges. In order to get upper bounds on the game chromatic number, He et al showed that every planar graph $G$ without leaves and 4cycles has $M$-degree at most 8 and gave an example of such a graph with $M$-degree 3. This yields upper bounds on the game chromatic number of $\mathrm{C}_{4}$-free planar graphs. We determine the maximum possible $M$-degrees for
\end{abstract}

Contract grant sponsor: RFBR; Contract grant numbers: 08-01-00673, 06-01-00694; Contract grant sponsor: NSF; Contract grant numbers: DMS-0400498, DMS0650784, DMS-0652306.

Journal of Graph Theory

(c) 2008 Wiley Periodicals, Inc. 
planar, projective-planar and toroidal graphs without leaves and 4-cycles. In particular, for planar and projective-planar graphs this maximum is 7 . (C) 2008 Wiley Periodicals Inc. J Graph Theory 60: 80-85, 2009

Keywords: planar graphs; decomposition; degree; game coloring

\section{INTRODUCTION}

For an edge $x y$ in a graph $G$, the maximum degree (for short, $M$-degree) $M(x y)$ is the maximum of the degrees of $x$ and $y$. The minimax degree (M-degree) of a graph $G$ is $M^{*}(G)=\min \{M(x y) \mid x y \in E(G)\}$. Let $\delta(G)$ and $\Delta(G)$ denote the minimum and maximum degrees of a graph $G$, respectively.

Kotzig [4] proved that $M^{*}(G) \leq 5$ for every triangle-free planar graph $G$ with $\delta(G) \geq 3$. Borodin [1,2] showed that $M^{*}(G) \leq 10$ for every planar graph $G$ with $\delta(G) \geq 3, M^{*}(G) \leq 7$ for every planar graph $G$ with $\delta(G) \geq 4$, and $M^{*}(G) \leq 6$ for every planar graph $G$ with $\delta(G) \geq 5$. All these bounds are tight.

The $M$-degree of planar graphs with $\delta(G) \geq 2$ is not bounded from above. For example, $M^{*}\left(K_{2, n}\right)=n$. Note that every cycle in $K_{2, n}$ has length 4 . He et al. [3] found upper bounds on $M$-degrees of planar graphs with $\delta(G) \geq 2$ and restrictions on girth. They used these bounds to estimate from above the game chromatic number and the game coloring number of such graphs. In particular, one of the main results in [3], Theorem 2.2, says that $M^{*}(G) \leq 8$ for planar graphs $G$ having no leaves and 4 -cycles. This result yields that every $C_{4}$-free planar graph can be decomposed into a forest and a graph with maximum degree at most 7 , which in turn implies that the game chromatic number and the game coloring number of every $C_{4}$-free planar graph is at most 11 . It is also mentioned in [3] that it is not known whether 8 is the exact bound and that the $M$-degree of dodecahedron is 3 .

In this note we determine the exact upper bounds on $M$-degrees for $C_{4}$-free graphs $G$ with $\delta(G) \geq 2$ embeddable into the plane, the projective plane, the torus and the Klein bottle.

Theorem 1. Let $G$ be a graph without 4-cycles and leaves. If $G$ is planar or projective-planar, then $M^{*}(G) \leq 7$. If $G$ is embeddable into the torus or the Klein bottle, then $M^{*}(G) \leq 8$. Both bounds are sharp.

As explained in [3], this implies

Corollary 1. If $G$ is a planar graph without 4-cycles, then

(i) G has an edge-partition into a forest and a subgraph $H$ with $\Delta(H) \leq 6$;

(ii) the game chromatic number and the game coloring number of $G$ is at most 10. 
Let $N(S)$ denote the Euler characteristic of a surface $S$. Recall that $N(S)=2$ if $S$ is the plane, $N(S)=1$ if $S$ is the projective plane, and $N(S)=0$ if $S$ is the torus or the Klein bottle; for the other surfaces $N(S)<0$.

From the proof of Theorem 1, it is easy to deduce the following theorem.

Theorem 2. Let G be a graph without 4-cycles and leaves embedded into a surface $S$, where $N(S)<0$. If $G$ has more than $-72 N(S)$ edges, then $M^{*}(G) \leq 8$.

Thus, a large graph on a fixed surface $S$ with $N(S)<0$ behaves in terms of $M^{*}$ as a graph embeddable into the torus or the Klein bottle.

\section{CONSTRUCTIONS}

For $k \geq 5$, a $k$-belt $B_{k}$ is the graph obtained from two disjoint $k$-cycles $\left(v_{1}, \ldots, v_{k}\right)$ and $\left(u_{1}, \ldots, u_{k}\right)$ by adding, for every $i=1, \ldots, k$, paths $\left(v_{i}, x_{i}, u_{i}\right),\left(v_{i}, y_{i}, u_{i+1}\right)$, $\left(v_{i}, w_{i}, v_{i+1}\right)$ and $\left(u_{i}, z_{i}, u_{i+1}\right)$ (the summation of subscripts taken modulo $k$ ). Graph $B_{6}$ is depicted below.

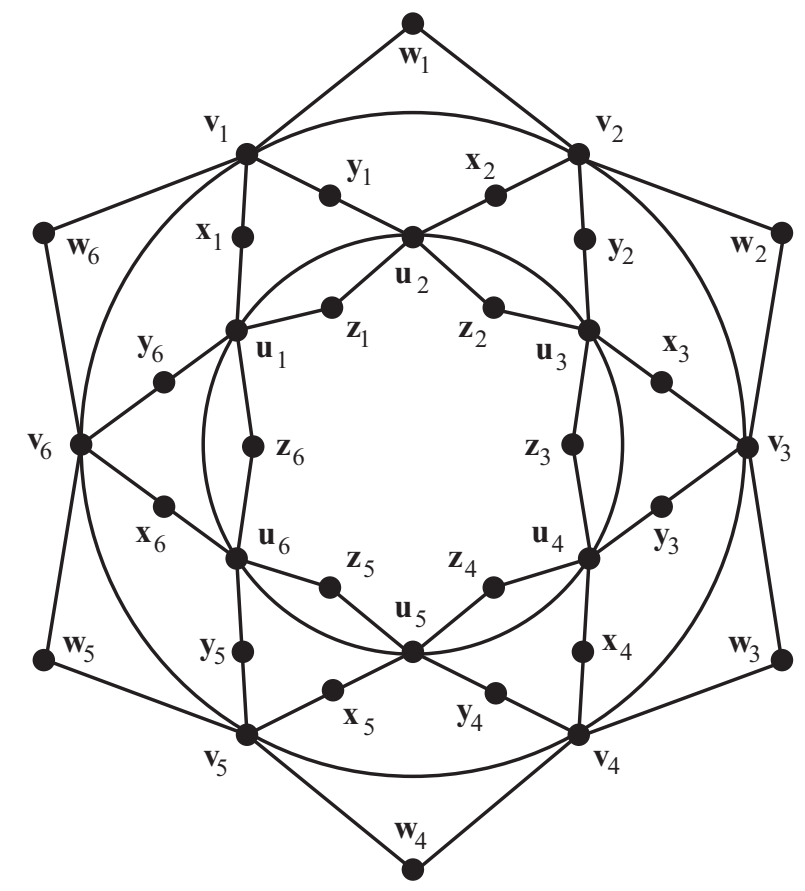

For $k \geq 5$ and $m \geq 2$, an $m$-multiple $k$-belt $B(k, m)$ is the graph obtained from $m$ disjoint copies $B_{k}^{j}$ of a $k$-belt $B_{k}$ by identifying for every $j=1, \ldots, m-1$ and every $i=1, \ldots, k$, vertex $u_{i}^{j}$ in $B_{k}^{j}$ with vertex $v_{i}^{j+1}$ in $B_{k}^{j+1}$ and vertex $z_{i}^{j}$ with vertex $w_{i}^{j+1}$. Graph $B_{k}$ can be also considered as $B(k, 1)$.

By the construction of $B(k, m)$, 
(b1) the degree of $v_{i}^{1}$ and of $u_{i}^{m}$ in $B(k, m)$ is $6,(i=1, \ldots k)$;

(b2) for $j=2, \ldots, m$, the degree of $v_{i}^{j}$ in $B(k, m)$ is $8,(i=1, \ldots k)$;

(b3) $B(k, m)$ has no 4-cycles (if $k \geq 5$ ).

Extremal examples for Theorem 1 can be easily obtained from $B(k, m)$. Indeed, let graph $X(k)$ be obtained from $B_{k}$ by adding a vertex $p$ connected by internally disjoint paths of length two with each $v_{i}(i=1, \ldots, k)$ and a vertex $q$ connected by internally disjoint paths of length two with each $u_{i}(i=1, \ldots, k)$. For $k \geq 5$, by (b3), graph $X(k)$ has no 4-cycles. For $k \geq 7$, by (b1) and (b2), $M^{*}(X(k))=7$. Since $X(k)$ is planar, it shows that the bound of Theorem 1 for planar and projective-planar graphs is tight (if true).

Similarly, for any $k \geq 5$ and $m \geq 5$, let $Y(k, m)$ be obtained from $B(k, m)$ by identifying for $i=1, \ldots, k$ vertex $v_{i}^{1}$ with vertex $u_{i}^{m}$ and vertex $w_{i}^{1}$ with vertex $z_{i}^{m}$. Then $M^{*}(Y(k, m)=8$ and again $Y(k, m)$ has no 4-cycles. Clearly, $Y(k, m)$ is embeddable into the torus. In order to get an analogous graph embeddable into the Klein bottle, we will identify $v_{i}^{1}$ not with $u_{i}^{m}$ but with $u_{k+1-i}^{m}$ and identify $w_{i}^{1}$ with $z_{k-i}^{m}$.

\section{UPPER BOUNDS}

Let $G$ be a counterexample to Theorem 1 with the fewest vertices. Let $k=7$ if $G$ is projective-planar and $k=8$ if $G$ is not projective-planar, but embeddable into the torus or the Klein bottle. Fix some such embedding of $G$ and let $F(G)$ be the set of faces of this embedding. Let $d(y)$ denote the degree of a vertex $y$ or of a face $y$. Call a vertex $v$ senior, if $d(v) \geq k+1$. By the choice of $G, M^{*}(G) \geq k+1$, and hence

Each closed walk $\left(w_{1}, \ldots, w_{t}\right)$ encounters senior vertices at least $\left\lceil\frac{t}{2}\right\rceil$ times.

Recall that the same vertex can be encountered several times on a closed walk.

Claim 1. G is connected.

Proof. Suppose that $G$ is the vertex-disjoint union of graphs $G_{1}$ and $G_{2}$, and there are no edges between $V\left(G_{1}\right)$ and $V\left(G_{2}\right)$. Let $G^{\prime}$ be obtained from $G$ by identifying a vertex $v \in V\left(G_{1}\right)$ with a vertex $w \in V\left(G_{2}\right)$. Since $G$ does not have leaves and 4-cycles, $G^{\prime}$ also does not. By the minimality of $G, M^{*}\left(G^{\prime}\right) \leq k$. But then $M^{*}(G) \leq k$, as well.

Since $G$ is connected, the boundary of every face is a closed walk and hence (1) applies to it.

Claim 2. For every vertex $v$, the total number of incident triangular faces and 2-vertices is at most $d(v)$.

Proof. Suppose that the neighbors of $v$ in the clockwise direction are $w_{1}, \ldots, w_{t}$. Let $f_{i}$ be the face containing walk $\left(w_{i}, v, w_{i+1}\right), i=1, \ldots, t$ (some 
$f_{i} \mathrm{~s}$ and/or $w_{i} \mathrm{~s}$ can coincide). If $f_{i}$ is a triangle, then since $M^{*}(G) \geq k+1$, at least one of $w_{i}$ and $w_{i+1}$ is senior; call such a vertex $x_{i}$. Since $G$ has no 4-cycles, all $x_{i} \mathrm{~s}$ are distinct. This yields the claim.

We now use discharging to obtain a contradiction with the assumption $M^{*}(G) \geq$ $k+1$.

Let the initial charge of every $x \in V(G) \cup F(G)$ be $\mu(x)=d(x)-4$. By Euler's formula $|V(G)|-|E(G)|+|F(G)| \geq N(S)$, we have

$$
\sum_{x \in V(G) \cup F(G)}(d(x)-4)=\sum_{x \in V(G) \cup F(G)} \mu(x) \leq-4 N(S) .
$$

The vertices and faces of $G$ discharge their initial charge by the following rules:

Rule 1. Every non-triangular face gives $1 / 2$ to each incident vertex of degree 2 or 3.

Rule 2. Every non-triangular face gives additional $1 / 2$ to each incident vertex $v$ of degree 2, such that the other face incident with $v$ is a triangle.

Rule 3. Every senior vertex gives $1 / 2$ to each adjacent 2 -vertex and each incident triangular face.

It remains to show that the final charge $\mu^{*}(y)$ is nonnegative for each $y \in V(G) \cup$ $F(G)$, and that the final charge of every vertex of degree at least 9 is strictly positive. This yields a contradiction to (2), since the total charge does not change, and hence should be strictly negative when $G$ is projective-planar and non-positive when $G$ is embeddable into the torus or the Klein bottle.

If $y$ is a triangular face, then by (1) it has at least two senior vertices incident to it, and it gets from them $1 / 2+1 / 2$ by Rule 3; so $\mu^{*}(y) \geq 3-4+2 \times 1 / 2=0$.

If $y$ is a face with $d(y) \geq 7$, then by (1), it encounters at most $\lfloor d(y) / 2\rfloor$ incident non-senior vertices, and hence by Rules 1 and $2, \mu^{*}(y) \geq d(y)-4-\lfloor d(y) / 2\rfloor \geq 0$.

Suppose $y$ is a 5 -face $\left(v_{1}, \ldots, v_{5}\right)$. Again by (1), it has at most two incident non-senior vertices. Moreover, neither of these vertices is a 2 -vertex incident with a triangular face, since if a vertex $v_{i}$ would be such a 2-vertex, then $v_{i-1} v_{i+1} \in E(G)$, and $G$ has a 4-cycle containing this edge. Thus, Rule 2 is not applicable to $y$, and $\mu^{*}(y) \geq 5-4-2(1 / 2)=0$.

Similarly, if $y$ is a 6-face, then it has at most three incident non-senior vertices, and at most one of these non-senior vertices is a 2-vertex incident with a triangular face. So, $\mu^{*}(y) \geq 6-4-3(1 / 2)-1 / 2=0$.

Suppose now that $y$ is a vertex.

If $d(y)=2$, then by Rule 3 it gets $2(1 / 2)=1$ from the adjacent vertices and by Rules 1 and 2, exactly 1 from the incident faces. Hence, $\mu^{*}(y)=2-4+1+1=0$.

If $d(y)=3$, then since $G$ has no 4-cyles, by Rule 1 , it gets at least $2(1 / 2)=1$ from the incident non-triangular faces. Hence, $\mu^{*}(y) \geq 3-4+1=0$. 
If $4 \leq d(y) \leq 7$, then $v$ gives out nothing, and $\mu^{*}(y)=d(y)-4 \geq 0$.

If $d(y)=8$, then by Rule 3 and Claim $2, \mu^{*}(y) \geq d(y)-4-d(y) / 2=0$. Finally, if $d(y) \geq 9$, then for the same reasons, $\mu^{*}(y) \geq d(y)-4-d(y) / 2>0$.

This completes the proof of Theorem 1.

\section{DEDUCING THEOREM 2}

Let $G$ be a counterexample to Theorem 2. In particular, this means that every edge is incident with a vertex of degree at least 9 (a senior vertex). Consider the discharging from the previous section. Then, as shown a few lines above, each senior vertex $v$ in $G$ has $\mu^{*}(v) \geq d(v)-4-d(v) / 2=(d(v)-8) / 2 \geq 1 / 2$. For each senior vertex $v$, we distribute $\mu^{*}(v)$ evenly among the edges incident with $v$.

Let $v(x)$ denote the new charge of an element $x \in V(G) \cup E(G) \cup F(G)$. Then $v(e) \geq(d(v)-8) / 2 d(v) \geq 1 / 18$ for every edge $e \in E(G), v(f)=\mu^{*}(f) \geq 0$ for every $f \in F(G)$, and $v(v)=0$ for every $v \in V(G)$. Now (2) implies

$$
|E(G)| / 18 \leq \sum_{x \in E(G) \cup F(G)} v(x)=\sum_{x \in V(G) \cup F(G)} \mu^{*}(x) \leq-4 N(S),
$$

which contradicts the assumption that $|E(G)|>-72 N(S)$.

\section{ACKNOWLEDGMENTS}

The research was partially supported by the RFBR grants 05-01-00816 and 06-0100694 (to O.V.B.) and NSF grants DMS-0400498 and DMS-0650784 (to A.V.K.) and DMS-0652306 (to G.Y.).

\section{REFERENCES}

[1] O. V. Borodin, A generalization of Kotzig's theorem and prescribed edge coloring of plane graphs, Math Notes Acad Sci USSR 48 (1990), 1186-1190 (in Russian).

[2] O. V. Borodin, Structural theorem on plane graphs with application to the entire coloring, J Graph Theory 23 (1996), 233-239.

[3] W. He, X. Hou, K. W. Lih, J. Shao, W. Wang, and X. Zhu, Edge-partitions of planar graphs and their game coloring numbers, J Graph Theory 41 (2002), 307-317.

[4] A. Kotzig, Contribution to the theory of Eulerian polyhedra, Mat Čas 13 (1963), 20-34 (in Russian). 